\title{
Novel progranulin mutations with reduced serum-progranulin levels in frontotemporal lobar degeneration
}

\author{
Huei-Hsin Chiang ${ }^{1}$, Charlotte Forsell ${ }^{1}$, Lena Lilius ${ }^{1}$, Linn Öijerstedt ${ }^{1}$, Steinunn Thordardottir ${ }^{1,2}$, \\ Krishnan Shanmugarajan ${ }^{1}$, Marie Westerlund ${ }^{1}$, Inger Nennesmo ${ }^{3}$, Håkan Thonberg ${ }^{2}$ and Caroline Graff $f^{\star, 1,2}$ \\ Frontotemporal lobar degeneration (FTLD) is a progressive neurodegenerative disease with an age at onset generally below \\ 65 years. Mutations in progranulin (GRN) have been reported to be able to cause FTLD through haploinsufficiency. \\ We have sequenced GRN in 121 patients with FTLD and detected six different mutations in eight patients: p.Gly35Glufs*19, \\ p.Asn 118Phefs*4, p.Val200Glyfs* 18 , p.Tyr294*, p.Cys $404^{*}$ and p.Cys416Leufs*30. Serum was available for five of the \\ mutations, where the serum-GRN levels were found to be $>50 \%$ reduced compared with FTLD patients without GRN \\ mutations. Moreover, the p.Cys416Leufs ${ }^{*} 30$ mutation segregated in an affected family with different dementia diagnoses. \\ The mutation frequency of GRN mutation was $6.6 \%$ in our FTLD cohort. \\ European Journal of Human Genetics (2013) 21, 1260-1265; doi:10.1038/ejhg.2013.37; published online 6 March 2013
}

Keywords: GRN; FTLD; progranulin ELISA

\section{INTRODUCTION}

Frontotemporal lobar degeneration (FTLD) is a heterogeneous neurodegenerative disease affecting individuals primarily below the age of 65 years. ${ }^{1-3}$ Common clinical symptoms are progressive dysfunction of behavior and/or language. A subgroup of FTLD patients develops the motor neuron disease amyotrophic lateral sclerosis (ALS). ${ }^{2-4} \mathrm{Up}$ to $50 \%$ of FTLD patients have a positive family history for dementia, indicating that genetic factors contribute to the etiology. Mutations in several genes have been shown to cause FTLD and most frequently in the microtubule-associated protein tau $^{5}$ (MAPT, OMIM \#600274), progranulin 6 ,7 (GRN, OMIM \#607485) and in the recently identified chromosome 9 openreading frame 72 gene $^{8,9}$ (C9orf72, OMIM \#105550). Mutations in GRN account for $5-10 \%$ of all the FTLD cases. ${ }^{10,11}$ Deletions of one copy of GRN have been reported, ${ }^{12,13}$ but the majority of mutations result in null alleles, leading to an approximately $50 \%$ reduction of the precursor protein GRN, which can be measured in serum, plasma and CSF. ${ }^{14-16}$ Mutations in GRN have been reported in different clinical phenotypes including Alzheimer disease $(\mathrm{AD})$ and corticobasal syndrome (CBS). ${ }^{17-20}$ In FTLD-ALS and ALS patients, the role of $G R N$ is unclear because only missense variations with unclear pathogenicity have been detected. ${ }^{21,22}$ In order to investigate the GRN-mutation frequency in FTLD patients recruited from an outpatient memory clinic in Stockholm, Sweden, we have sequenced GRN and measured serum-GRN levels.

\section{MATERIALS AND METHODS}

Subjects

DNA from 121 patients clinically diagnosed with FTLD was collected at the Department of Geriatric Medicine, Karolinska University Hospital. The 121
FTLD patients were diagnosed according to Neary et al. ${ }^{3}$ The clinical and neuropathological subdiagnoses of the patients are specified in Table 1. Patients with FTLD-ALS were included because FTLD and ALS could be considered to be part of the same disease spectrum. To perform segregation analysis, DNA was collected from available blood relatives. Serum for measuring GRN levels was available from 76 out of 121 patients, as well as from four relatives to mutation carriers.

The control population consisted of 171 neurologically healthy individuals between 72 and 85 years from the Swedish National Study on Aging and Care (http://www.snac-k.se/), with a mini mental-state examination score $\geq 28$.

Informed consent from all participants and approval from the local ethics committee in Stockholm was obtained.

\section{Genetic analyses}

GRN sequencing and haplotype analysis. All 13 exons and $\sim 60$ nucleotides into the flanking intronic sequences in GRN were PCR amplified and sequenced using in-house and previously published primers. ${ }^{6,7}$ For haplotype analyses, microsatellites and single-nucleotide polymorphisms (SNPs) in GRN were used (Table 2). The SNP genotypes were obtained from GRN sequence (A). All sequences and microsatellites were run on an ABI 3100 Genetic Analyzer (Applied Biosystems, Foster City, CA, USA). The primer sequences and PCR conditions are available upon request.

GRN copy-number assay. The possibility of a GRN gene deletion was inferred from GRN SNP genotypes, that is, heterozygous patients were assumed not to have a deletion. In addition, for patients who were homozygous for all GRN SNPs in Table 2, the serum-GRN levels were used to determine if reduced protein levels were a consequence of haploinsufficiency, which could serve as an indicator for a GRN deletion. For patients who were homozygous for all GRN SNPs and had no serum available, either TaqMan (Applied Biosystems, Carlsbad, CA, USA) copy-number assay (B) or multiplex ligation-dependent probe amplification (MLPA) assays were used. Quantitative real-time PCR was

${ }^{1}$ Department of NVS, Karolinska Institutet, KI-Alzheimer Disease Research Center, Stockholm, Sweden; ${ }^{2}$ Genetics Unit, Department of Geriatric Medicine, Karolinska University Hospital, Stockholm, Sweden; ${ }^{3}$ Division of Pathology, Department of Laboratory Medicine, Karolinska University Hospital, Stockholm, Sweden

${ }^{*}$ Correspondence: Professor C Graff, Department NVS, Karolinska Institutet, KI-Alzheimer Disease Research Center, Novum level 5, Stockholm, SE-141 86, Sweden. Tel: + 468 58583619; Fax: + 468 58583610; E-mail: caroline.graff@ki.se

Received 3 September 2012; revised 5 February 2013; accepted 6 February 2013; published online 6 March 2013 
Table 1 Clinical and neuropathological subgroups in the FTLD study cohort

\begin{tabular}{llr}
\hline Clinical subgroups & Total cases: 121 & $\mathrm{~N}$ \\
\hline & FTLD & 40 \\
& bvFTD $^{\text {a }}$ & 30 \\
& PPA $^{\text {b }}$ & 7 \\
SD & 18 \\
& PNFA & 9 \\
& FTLD-ALS & 9 \\
& Dementia with frontal signs & 8 \\
& Total cases: 21 & $\mathrm{N}$ \\
Neuropathology & & 1 \\
& FTLD & 14 \\
& FTLD-TDP & 5 \\
& FTLD-MND & 1
\end{tabular}

Abbreviations: bvFTD, behavior variant FTLD; FTLD, frontotemporal lobar degeneration; FTLD-ALS, FTLD with amyotrophic lateral sclerosis; FTLD-MND, FTLD with motor neuron disease; FTLD-ni, FTLD with no inclusions; FTLD-TDP, FTLD with TDP-43-positive inclusions; PNFA, progressive non-fluent aphasia; PPA, primary progressive aphasia; SD, semantic dementia.

Clinical subgroup not specified.

'Not able to distinguish between SD and PNFA.

'Unspecified dementia with behavioral symptoms.

${ }^{\mathrm{d}}$ Tau-negative. Very few pospho-TDP-43 and p62 immunoreactive neuronal inclusions, which could be because of long fixation time (six months).

Table 2 GRN SNPs and microsatellites investigated in FTLD patients

\begin{tabular}{lc}
\hline Genetic marker & Genomic start position \\
\hline D17S932 & 41204731 \\
D17S1789 & 41752986 \\
D17S951 & 41820093 \\
rs17523519 & 42422380 \\
rs3859268 & 42423263 \\
rs9897526 & 42426940 \\
rs34424835 & 42427549 \\
rs25646 & 42427630 \\
rs850713 & 42427732 \\
rs72824736 & 42428538 \\
rs5848 & 42430244 \\
D17S930 & 42723441 \\
D17S1861 & 42807013 \\
D17S934 & 43057680 \\
D17S1868 & 47184853 \\
D17S790 & 52798868 \\
D17S792 & 58239779 \\
D17S944 & 61436306 \\
\hline
\end{tabular}

The SNPs in GRN (in gray) were used to evaluate GRN gene deletion. For haplotype analysis, the SNPs were also used together with the microsatellites flanking GRN.

performed with TaqMan copy-number assays and analyzed on an ABI 7500 Fast real-time PCR system according to the manufacturer's protocol. The assays amplify target regions within the GRN gene in exon 1 (custom design), exon 3 (custom design), exon 12 (assay ID: Hs02250283_cn) and exon 13 (assay ID: Hs00336119_cn). TaqMan copy-number reference assay RNase P was used as a calibrator in every reaction together with the target assay-primers in a duplex real-time PCR. MLPA assays were performed using SALSA MLPA kit P275-B1 MAPT-PGRN (MRC-Holland, Amsterdam, the Netherlands) according to the manufacturer's protocol.

Serum-GRN measurements. GRN levels were determined by the Progranulin (human) ELISA Kit (Adipogen, Incheon, Korea) according to the manufacturer's protocol in duplicate with a dilution of 1:50 of the serum. Normal control samples as well as a positive control for a pathogenic GRN mutation resulting in haploinsufficiency were included in all ELISA experiments.

\section{Bioinformatics}

The Alamut software (Interactive Biosoftware, www.interactive-biosoftware. com) was used to analyze the effect of detected genetic variations. The software integrates five splice site prediction programs: SpliceSiteFinder, MaxEntScan, Human Splice Finder, NNSPLICE and GeneSplicer. For variations affecting the amino-acid sequence, Alamut uses the prediction programs: PolyPhen-2, Align GVGD and SIFT.

\section{Histology and immunohistochemistry}

Paraffin-embedded formalin-fixed sections from the frontal, parietal and temporal cortex and the hippocampus were analyzed on 21 FTLD patients with the following antibodies: anti-ubiquitin (1:1000; DakoCytomation, Glostrup, Denmark), anti-phospho-TAR DNA-binding protein 43 (TDP-43) (1:20 000; Cosmo Bio Co Ltd, Tokyo, Japan) and anti-tau (AT8 1:500; Innogenetics, Ghent, Belgium). Staining with hematoxylin and eosin, luxol fast blue and Bielschowsky's silver stain were also performed.

\section{RESULTS}

\section{Genetic analysis}

Sequencing of GRN in 121 FTLD patients identified several common SNPs (Table 2). Furthermore, nine rare variations were indentified: one intronic variation; two missense variations; and six variations introducing premature stop codons, of which two were frameshift, two splice site variations and two nonsense variations (Table 3). Of the identified rare variations, only p.Arg433Trp (numbering according to NP_002078.1 with methionine as amino acid 1) was seen in controls, with a frequency of $1 / 171$ (Table 3 ).

The p.Gly35Glufs ${ }^{\star} 19$ mutation was detected in patient A01 who before the age of 59 years experienced memory and language dysfunction and was diagnosed with primary progressive aphasia (Table 4). This mutation has previously been detected in the Swedish Karolinska FTLD family. ${ }^{23}$ Haplotype analysis in the two families showed that patient A01 and the Swedish Karolinska family shared at the most an $\sim 297 \mathrm{kbp}$ disease haplotype, spanning from the mutation downstream to the microsatellite marker D17S930. However, they did not share the same allele at rs3859268 located 883 bp centromeric to the mutation.

The second frameshift variation p.Cys416Leufs ${ }^{\star} 30$ was detected in patient G01 who at the age of 64 years developed memory and language dysfunction. Patient G01 had initially received a clinical diagnosis of AD but was diagnosed as FTLD with TDP-43 immunoreactivity (FTLD-TDP) after neuropathological examination. Segregation analysis in the family was performed where six family members were affected with different types of neurological conditions, such as FTLD and AD with vascular dementia (VaD), and 11 members were without any neurological symptoms. The variation was present in four and absent in two of the six affected family members. One of the affected member without the frameshift variation was diagnosed with multiple sclerosis (MS) and the other with FTLD (Supplementary Text, patient G06). Among the 11 healthy relatives, p.Cys416Leufs ${ }^{\star} 30$ was absent in eight, and three of those eight healthy relatives were above the age of 75 years. The mean age at onset for the affected carriers was 65 years (range 55-71 years). Of the three unaffected carriers, one was above the mean age at onset and the other two were under the age at onset, showing a variable age at onset which is in agreement with previous reports (Supplementary Figure 1). $7,18,23-25$

One splice site variation c. $462+1 \mathrm{G}>\mathrm{C}$ (numbering according to NM_002087.2, starting with A in ATG as nucleotide position 1) was 
Table 3 Detected GRN variations in FTLD patients and controls

\begin{tabular}{|c|c|c|c|c|c|c|c|c|c|}
\hline \multirow[b]{2}{*}{$c D N A^{\mathrm{b}}$} & \multirow[b]{2}{*}{ Protein $^{c}$} & \multirow[b]{2}{*}{ Function } & \multirow[b]{2}{*}{ Location } & \multirow[b]{2}{*}{$S N P I D$} & \multicolumn{3}{|c|}{ Present in ${ }^{\mathrm{a}}$} & \multirow[b]{2}{*}{$\begin{array}{c}\text { Novel } \\
\text { Variation }\end{array}$} & \multirow[b]{2}{*}{ Mode of action } \\
\hline & & & & & $M A F^{\mathrm{d}}$ & $\begin{array}{c}\text { FTLD } \\
(\mathrm{n}=100)\end{array}$ & $\begin{array}{c}\text { Control } \\
(\mathrm{n}=171)\end{array}$ & & \\
\hline c.102delC & p.Gly35Glufs*19 & Frameshift & Exon 2 & rs63751073 & & 1 & 0 & No & Nonsense-mediated mRNA decay \\
\hline c. $462+1 G>C$ & p.Asn118Phefs ${ }^{*} 4$ & Splice site & Intron 5 & & & 1 & 0 & Yes & Nonsense-mediated mRNA decay \\
\hline c. $463-25 C>T$ & & Intron & Intron 5 & & & 1 & 0 & Yes & Not damaging \\
\hline c. $1212 C>A$ & p.Cys404* & Nonsense & Exon 11 & & & 1 & 0 & Yes & Nonsense-mediated mRNA decay \\
\hline c.1246_1247ins T & p.Cys416Leufs*30 & Frameshift & Exon 11 & & & 1 & 0 & Yes & Nonsense-mediated mRNA decay \\
\hline c. $1294 C>T$ & p.Arg432Cys & Missense & Exon 11 & rs63750130 & & 1 & 0 & No & Possibly not damaging \\
\hline c. 1297 C > T & p.Arg433Trp & Missense & Exon 11 & rs63750412 & 0.003 & 1 & 1 & No & Possibly not damaging \\
\hline
\end{tabular}

a Number of individuals heterozygous for the identified genetic variation.

bThe numbering is according to NM_002087.2 starting with A in ATG (start codon) as nucleotide position 1.

'The numbering is according to NP_002078.1 with methionine as amino acid 1 .

dMinor allele frequency (MAF) in 1000 Genome.

epredicted by the Alamut software.

Table 4 Patients with potentially pathogenic variations in GRN

\begin{tabular}{|c|c|c|c|c|c|c|c|}
\hline Patient ID & Protein $^{\text {a }}$ & $\begin{array}{c}\text { Age at onset } \\
\text { (years) }\end{array}$ & $\begin{array}{l}\text { Age at death } \\
\text { (years) }^{\mathrm{b}}\end{array}$ & Clinical subgroup & Neuropathological diagnosis & Family historyc & $\begin{array}{c}\text { Serum GRN } \\
(n g / m l)\end{array}$ \\
\hline A01 & p.Gly35Glufs*19 & Before 59 & - & PPA & & Yes & 31.1 \\
\hline B01 & p.Asn118Phefs*4 & 57 & - & bvFTD & & Possible family history & 45.8 \\
\hline $\mathrm{CO1}$ & p.Val200Glyfs*18 & $58-59$ & - & bvFTD & & No information & 51.6 \\
\hline D01 & p.Tyr294* & 54 & 58 & PNFA & FTLD-TDP & Yes & 42.2 \\
\hline E01 & p.Tyr294* & $69-70$ & 76 & bvFTD & & Yes & 44.0 \\
\hline E02 & p.Tyr294* & 61 & 66 & bvFTD & & Yes & 41.8 \\
\hline F01 & p.Cys404* & 58 & 66 & bvFTD & & No information & \\
\hline G01 & p.Cys416Leufs*30 & 64 & 71 & $A D$ & FTLD-TDP & Yes & $46.7^{d}$ \\
\hline $\mathrm{HO1}$ & p.Arg432Cys & 58 & 69 & FTLD/AD with parkinsonism & & No information & 117.7 \\
\hline
\end{tabular}

The numbering is according to NP_002078.1 with methionine as amino acid 1.

bThe information is current as of February 2012.

'Positive family history is defined as having dementia in at least one first-degree relative or at least one second-degree relative (including half siblings).

${ }^{\mathrm{d}} \mathrm{GRN}$ level obtained from a family member with the same mutation.

detected in patient $\mathrm{B} 01$ who at the age of 57 years developed memory problems and language dysfunction. After examination by a neuropsychologist and speech therapist, the patient received the clinical subdiagnosis bvFTD (Table 4). The variation is located in the splice donor site of intron 5 and was predicted to lead to skipping of exon 5 and a premature stop codon, p.Asn $118 \mathrm{Phefs}^{\star} 4$.

The other splice site variation, c.708+1G $>$ A, was detected in patient $\mathrm{C} 01$ who at an age between 58 and 59 years developed memory problems, behavior changes and language dysfunction. Patient C01 received the FTLD diagnosis at the age of 63 years (Table 4). The variation is located in the splice donor site of intron 7 and was predicted to lead to skipping of exon 7 and a premature stop codon, p.Val200Glyfs ${ }^{\star} 18$.

The nonsense variation p.Tyr294* was identified in three patients (patients D01, E01 and E02) who had different clinical presentations of FTLD, as well as a 15-year range in age at onset (Table 4). Since DNA from patient D01's family members was not available, the haplotypes of patient D01 could not be phased. However, patient E01 and E02 were shown to be siblings. A possible disease haplotype for E01 and E02 could be determined, which showed that patient D01 possibly shared one allele from D17S789 to D17S934, an $\sim 1.3 \mathrm{Mbp}$ region, which indicated that all the three patients could be related.
The nonsense variation p.Cys404* was detected in patient F01 who developed memory difficulties and behavioral changes at the age of 58 years and was diagnosed as bvFTD (Table 4).

The intronic variation c.463-25C $>\mathrm{T}$ was suggested by in silico analysis to be non-pathogenic. Finally, the missense variations p.Arg432Cys (patient H01) and p.Arg433Trp were predicted by two out of three protein-structure prediction programs to not to be damaging.

The possibility of a complete deletion of one copy of the GRN gene was excluded in 114 out of 121 FTLD patients as they were heterozygous for one or more GRN SNPs and/or had serum-GRN levels above $86 \mathrm{ng} / \mathrm{ml}$ (two SD from the mean GRN level in non-mutation carriers). In the remaining seven patients, the GRN copy number was determined to be normal by MLPA or TaqMan copy-number assays. A more detailed description of the clinical history on all patients with potential pathogenic GRN mutations and their relatives is available in the Supplementary text.

\section{Serum-GRN levels}

In addition to the genetic analyses, 76 of the 121 FTLD patients had serum available for GRN level analyses. Moreover, serum from two members of the Karolinska family and two relatives to patient 
G01 (one carrier and a non-carrier from both families) were available, so in total 80 samples were analyzed. Eight serum samples had GRN levels lower than $50 \mathrm{ng} / \mathrm{ml}$ (mean $43.5 \pm 5.6 \mathrm{ng} / \mathrm{ml}$; range $31.9-51.6 \mathrm{ng} / \mathrm{ml}$ ) and were shown to carry premature stop codons when sequenced (Figure 1). These mutation carriers had $>50 \%$ lower GRN levels compared with the mean serum-GRN level in patients $(n=70)$ without pathogenic $G R N$ variations (mean $141.0 \pm 27.6 \mathrm{ng} /$ $\mathrm{ml}$ and range $84.5-220.8 \mathrm{ng} / \mathrm{ml}$ ). The mean serum-GRN levels in patients without GRN mutations were similar to the two healthy non-carriers, one from the Karolinska family $(123.8 \mathrm{ng} / \mathrm{ml})$ and one relative of patient G01 $(185.8 \mathrm{ng} / \mathrm{ml})$. The patients carrying missense variations, p.Arg432Cys and p.Arg433Trp, had seemingly normal GRN levels of 119.6 and $148.7 \mathrm{ng} / \mathrm{ml}$, respectively (Figure 1).

\section{Histology and immunohistochemistry}

Autopsy was available for two out of eight GRN-mutation carriers and 19 out of 113 non-mutation carriers. The neuropathological examinations of the two GRN-mutation carriers are described here. The formaldehyde-fixed brain of patient D01 (p.Tyr294*) weighed $1115 \mathrm{~g}$. The frontal lobes were atrophic, more pronounced on the left side. There was general atrophy of the parietal and temporal lobes, and microscopy showed prominent loss of cortical neurons in sections from the left frontal lobe with astrogliosis. The right frontal lobe was better preserved as were the temporal and parietal lobes. Immunohistochemistry demonstrated tau-negative, ubiquitin- and phospho-TDP-43-positive neuronal cytoplasmic inclusions (NCIs) and neuronal intranuclear inclusions (NIIs), as well as neurites predominantly in layer 2 of the cerebral cortex (Figures 2a-c). A few phospho-TDP-43-positive neuronal inclusions were observed in the granular cell layer of the dentate gyrus. Thus, patient D01 received the neuropathological diagnosis FTLD-TDP. ${ }^{1}$ According to the new harmonized FTLD-TDP neuropathological classification system, $G R N$-mutation carriers typically have type A histology predominantly in cortical layer 2 with numerous neurites, NCIs and few NIIs. Although the pathology was predominantly in the cortical layer 2, in agreement with type A histology, patient D01 had few phospho-TDP-43 immunoreactive neurites and NCIs, which is not typical for type A histology. 1,26,27

The formaldehyde-fixed brain of patient G01 (p.Cys416Leufs ${ }^{\star 30}$ ) weighed $630 \mathrm{~g}$. The gyri were severely atrophic, and microscopic examination showed widespread changes with loss of neurons, mainly in cortical layer 2, with vacuolation and astrogliosis. In the superficial layers of the cerebral cortex tau-negative, ubiquitin-positive and phospho-TDP-43-positive NCIs, NIIs and neurites were found (Figures $2 \mathrm{~d}-\mathrm{f}$ ). In the granular cell layer of the dentate gyrus, some phospho-TDP-43-positive NCIs were observed. Similarly to patient D01, patient G01 received the neuropathological diagnosis FTLDTDP with an atypical type A histology for the same reasons as described above for D01.,26,27

\section{DISCUSSION}

Since the discovery of GRN mutations in FTLD in 2006, there have been 69 pathogenic mutations reported in the Frontotemporal Dementia Mutation Database (http://www.molgen.vib-ua.be/FTD Mutations). We have in our screen detected six possible pathogenic variations in GRN in eight patients with different clinical presentations of FTLD. All of these variations were absent in our control individuals. Furthermore, the GRN levels in five out of the six identified premature stop-codon variations demonstrated a $>50 \%$ reduction in serum. The combined data indicate that the premature stop-codon variations are pathogenic. For p.Cys $404^{\star}$, no serum was available to demonstrate its consequence on the GRN levels, thus its pathogenicity still has to be proven. However, p.Cys404* results in a premature stop codon and was absent in 171 neurologically healthy controls (342 chromosomes) in support of its pathogenic nature.

Of the detected mutations, three have previously been reported by us and/or others: p.Gly35Glufs ${ }^{\star} 19$, p.Val200Glyfs ${ }^{\star} 18$ and p.Tyr294* (http://www.molgen.ua.ac.be/FTDMutations). ${ }^{18,20,23,24}$ The p.Gly35Glufs ${ }^{\star} 19$ identified in patient A01 has been reported in the Swedish Karolinska family, ${ }^{23}$ as well as in two FTLD patients, one from the United States and one from Sweden. ${ }^{18}$ The p.Gly35Glufs ${ }^{\star} 19$ mutation is a deletion of one out of four consecutive cytosines, and
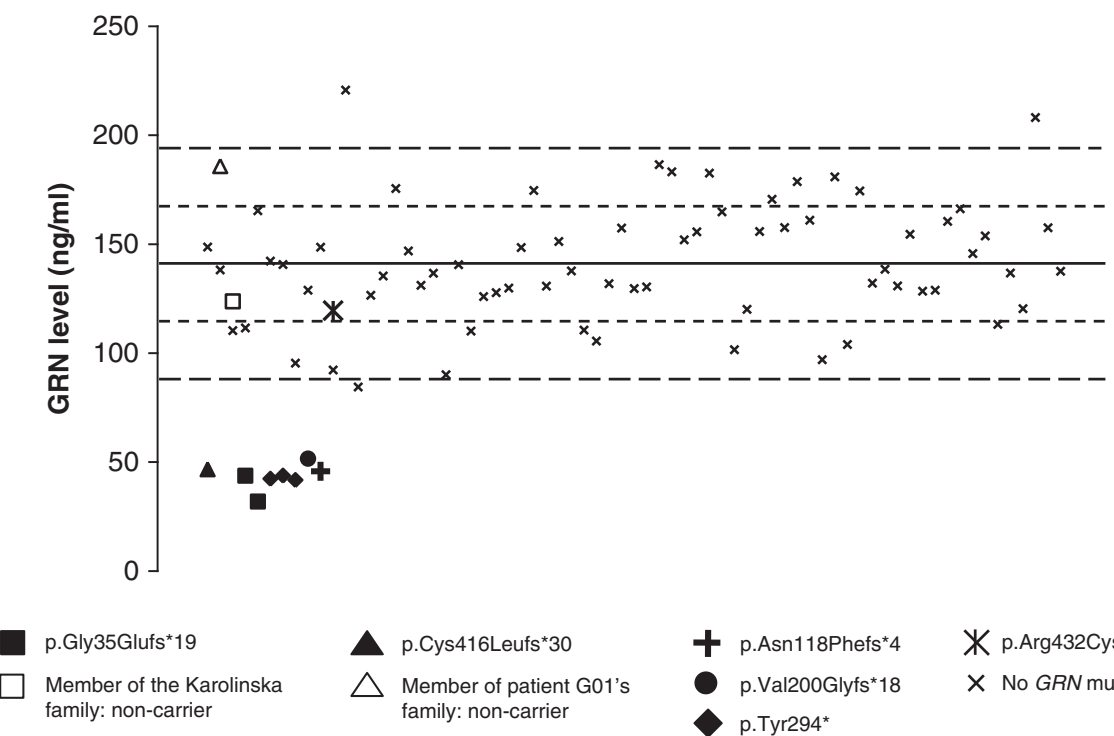

X p.Arg432Cys

$\times$ No GRN mutation

Figure 1 Serum-GRN levels in 76 FTLD patients and four additional family members with or without GRN mutations (Karolinska family: p.Gly35Glufs*19 carrier and non-carrier; G01 family: p.Cys416Leufs*30 carrier and non-carrier). The horizontal line indicates the mean serum-GRN level (141 ng/ml) of patients without premature stop codons $(n=70)$. The dashed lines represent one and two SD from the mean value, respectively. 

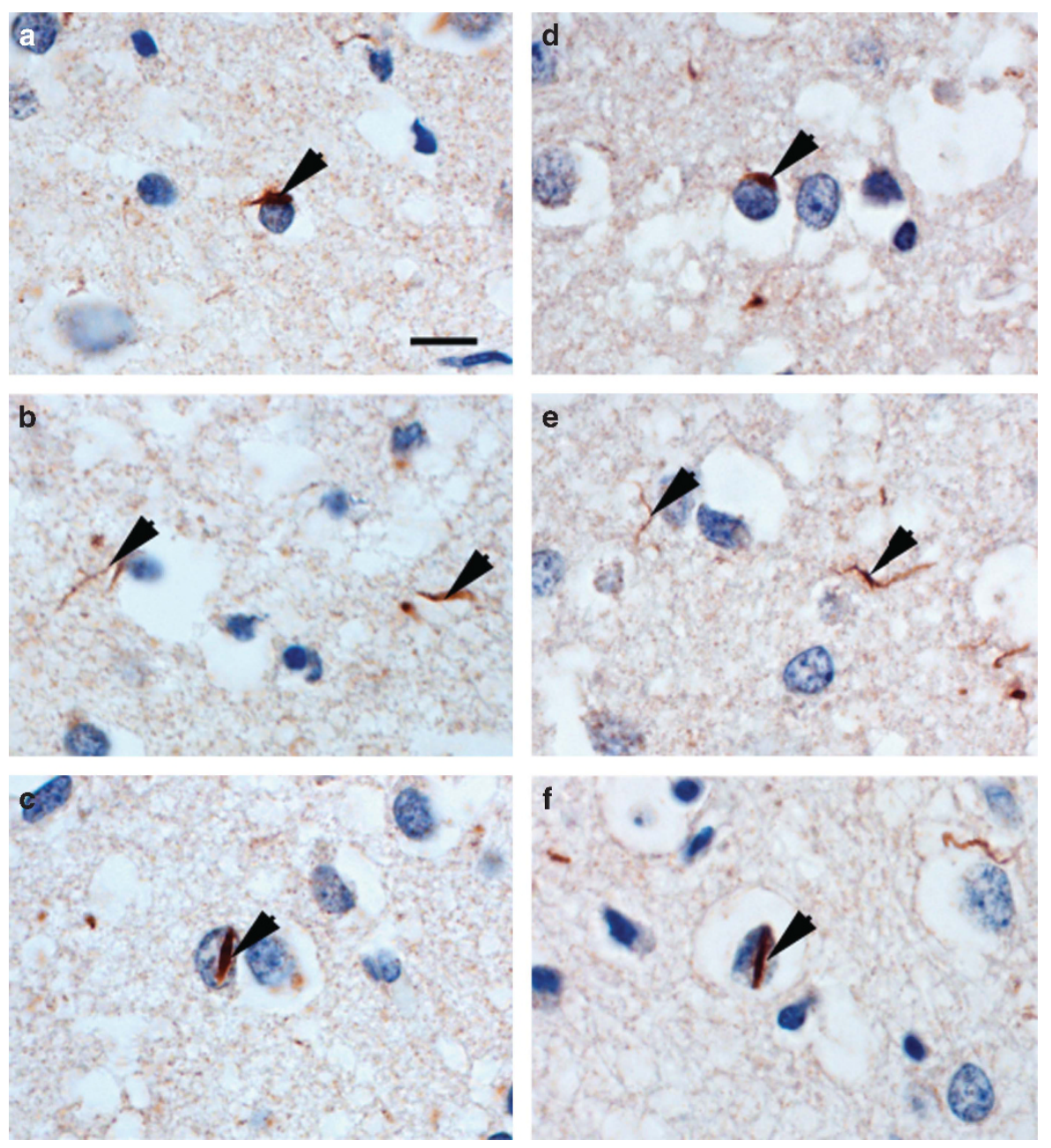

Figure 2 Immunohistochemical staining of the frontal cortex with phospho-TDP-43 antibody on patient D01 (a-c) and G01 (d-f). Phospho-TDP-43-positive $\mathrm{NCls}$ (arrows in figure $\mathbf{a}$ and $\mathbf{d}$ ), neurites (arrows in figure $\mathbf{b}$ and $\mathbf{e}$ ) and NIIs (arrows in figure $\mathbf{c}$ and $\mathbf{f}$ ) were detected in both patients. Scale bar $=20 \mu \mathrm{m}$.

three substitutions in this sequence have previously been reported, c. $99 \mathrm{C}>\mathrm{A}, \mathrm{c} .99 \mathrm{C}>\mathrm{T}$ and $\mathrm{c} .102 \mathrm{C}>\mathrm{T}$, indicating that this region is a possible hotspot for mutations. ${ }^{18,23-25,28}$ The family of patient A01 and the Karolinska family shared an $\sim 297 \mathrm{kbp}$ common disease haplotype as shown by comparing the disease haplotypes in the respective families. However, because they do not share a SNP (rs3859268) located $883 \mathrm{bp}$ away from the mutation, we suggest that the mutation has arisen twice or that the two families must be very distantly related. The p.Tyr $294^{*}$ was observed in three patients, two of which were found to be related. It was not possible to rule out that all three patients were related because we were unable to phase the haplotype in patient D01.

Segregation analysis was only possible for p.Cys416Leufs ${ }^{\star} 30$ that was detected in patient G01 who belonged to a family exhibiting different types of neurological phenotypes, such as FTLD and AD with $\mathrm{VaD}$. The analysis showed that the variation was present among the affected individuals except in two family members: one with MS and one who suffered from bipolar disorder from his 30s and later developed dementia and diagnosed with FTLD at the age of 81 years. There was no neuropathological confirmation of the FTLD diagnosis in this subject, and it is possible that the disease was a phenocopy or secondary to a life-long chronic psychiatric disease. The presence of p.Cys416Leufs ${ }^{\star} 30$ in affected family members with different dementia diagnoses indicates that the mutation can present itself with variable clinical pictures, a finding that is in agreement with previous reports. ${ }^{17-19}$

The two missense variations p.Arg432Cys and p.Arg433Trp have previously been reported in FTLD patients. ${ }^{14,18,24,25}$ According to the in silico analysis, codon 432 is weakly conserved but there is a large physicochemical difference between Arg and Cys. This variation has previously been reported in two FTLD patients and was absent in 646 control individuals. ${ }^{24,25}$ Furthermore, the substitution was predicted by in silico analysis to disturb the structure or the proteolytic cleavage of the precursor protein GRN and thus affect its biological function. ${ }^{25}$ In an in vitro study, it was observed that p.Arg432Cys resulted in reduced secretion of GRN. ${ }^{29}$ Moreover, there was a report of a patient with FTLD carrying p.Arg432Cys who had serum-GRN levels intermediate of controls and mutation carriers. ${ }^{16}$ However, this was not observed in patient $\mathrm{H} 01$ who had GRN levels similar to non-mutation carriers. Thus, the pathogenic nature of p.Arg432Cys is still unclear. The p.Arg433Trp has previously been reported to be non-pathogenic because it was detected in both patients and healthy controls and resulted in reference levels of GRN in serum and plasma. ${ }^{14,16}$ These results are in agreement with our findings and conclusion that p.Arg433Trp is not pathogenic.

There are reports on genetic factors that can modify age at onset for FTLD patients as well as GRN levels. The SNP rs1990622, 
located $6.9 \mathrm{kbp}$ downstream of the gene transmembrane protein $106 \mathrm{~B}$ (TMEM106B), has been shown to potentially modify both age at onset for GRN-mutation carriers and GRN levels in healthy individuals and GRN-mutation carriers. ${ }^{30-32}$ Furthermore, inconclusive results on $A P O E$ as a modifying factor for FTLD have been reported. ${ }^{17,18,33-36}$ It has also been speculated that rs5848 in the $3^{\prime}$ UTR of GRN is a binding site for the micro-RNA miR-659 and may regulate the translation of the GRN mRNA. ${ }^{37,38}$ Investigating these three modifying genes in our patient cohort did not reveal any association with age at onset or GRN levels in GRN-mutation carriers or the complete cohort. However, this could be due to the limited number of subjects investigated.

In summary, six GRN mutations were identified in eight patients and, where serum was available (serum was available for five of six mutations), a $>50 \%$ reduction of GRN levels was detected supporting their pathogenic nature. This corresponds to a mutation frequency of $6.6 \%(8 / 121)$ in our clinical series of FTLD patients. Furthermore, because there is a broad spectrum of clinical phenotypes in the mutation carriers, here clearly described for p.Cys416Leufs ${ }^{\star} 30$, inclusion of other neurological phenotypes is of importance when screening GRN.

\section{CONFLICT OF INTEREST}

The authors declare no conflict of interest.

\section{ACKNOWLEDGEMENTS}

We are thankful to the patients and their families included in this study. We would also thank Jenny Björkström and Anne Kinhult Ståhlbom for their help in compiling the clinical information, Vesna Jelic for patient referrals and Laura Fratiglioni for providing the control samples. This study was supported by Swedish Brain Power, Gun and Bertil Stohne's foundation, Gamla tjänarinnors foundation, Swedish Alzheimer foundation, Marianne and Marcus Wallenberg foundation, Knut and Alice Wallenberg foundation, Swedish Research Council, the King Gustaf V and Queen Victoria's Foundation of Freemasons and Karolinska Institutet doctoral-funding.

1 Mackenzie IR, Neumann M, Bigio EH et al: Nomenclature and nosology for neuropathologic subtypes of frontotemporal lobar degeneration: an update. Acta Neuropathol 2010; 119: 1-4.

2 McKhann GM, Albert MS, Grossman M, Miller B, Dickson D, Trojanowski JQ: Clinical and pathological diagnosis of frontotemporal dementia: report of the Work Group on Frontotemporal Dementia and Pick's Disease. Arch Neurol 2001; 58: 1803-1809.

3 Neary D, Snowden JS, Gustafson L et al: Frontotemporal lobar degeneration: a consensus on clinical diagnostic criteria. Neurology 1998; 51: 1546-1554.

4 Lomen-Hoerth C, Anderson T, Miller B: The overlap of amyotrophic lateral sclerosis and frontotemporal dementia. Neurology 2002; 59: 1077-1079.

5 Hutton M, Lendon CL, Rizzu P et al: Association of missense and $5^{\prime}$-splice-site mutations in tau with the inherited dementia FTDP-17. Nature 1998; 393: 702-705.

6 Baker M, Mackenzie IR, Pickering-Brown SM et al: Mutations in progranulin cause tau-negative frontotemporal dementia linked to chromosome 17. Nature 2006; 442: 916-919.

7 Cruts M, Gijselinck I, van der Zee J et al: Null mutations in progranulin cause ubiquitin-positive frontotemporal dementia linked to chromosome 17q21. Nature 2006; 442: 920-924.

8 Dejesus-Hernandez M, Mackenzie IR, Boeve BF et al: Expanded GGGGCC hexanucleotide repeat in noncoding region of C9ORF72 causes chromosome 9p-Linked FTD and ALS. Neuron 2011; 72: 245-256.

9 Renton AE, Majounie E, Waite A et al: A hexanucleotide repeat expansion in C9ORF72 is the cause of chromosome 9p21-Linked ALS-FTD. Neuron 2011; 72: 257-268.

10 Rohrer JD, Guerreiro R, Vandrovcova J et al: The heritability and genetics of frontotemporal lobar degeneration. Neurology 2009; 73: 1451-1456.
11 Seelaar H, Rohrer JD, Pijnenburg YA, Fox NC, van Swieten JC: Clinical, genetic and pathological heterogeneity of frontotemporal dementia: a review. J Neurol Neurosurg Psychiatry 2011; 82: 476-486.

12 Gijselinck I, van der Zee J, Engelborghs S et al: Progranulin locus deletion in frontotemporal dementia. Hum Mutat 2008; 29: 53-58.

13 Rovelet-Lecrux A, Deramecourt V, Legallic S et al: Deletion of the progranulin gene in patients with frontotemporal lobar degeneration or Parkinson disease. Neurobiol Dis 2008; 31: 41-45

14 Finch N, Baker M, Crook R et al: Plasma progranulin levels predict progranulin mutation status in frontotemporal dementia patients and asymptomatic family members. Brain 2009; 132: 583-591.

15 Ghidoni R, Benussi L, Glionna M, Franzoni M, Binetti G: Low plasma progranulin levels predict progranulin mutations in frontotemporal lobar degeneration. Neurology 2008; 71: 1235-1239.

16 Sleegers K, Brouwers N, Van Damme P et al: Serum biomarker for progranulinassociated frontotemporal lobar degeneration. Ann Neurol 2009; 65: 603-609.

17 Beck J, Rohrer JD, Campbell T et al: A distinct clinical, neuropsychological and radiological phenotype is associated with progranulin gene mutations in a large UK series. Brain 2008; 131: 706-720.

18 Gass J, Cannon A, Mackenzie IR et al: Mutations in progranulin are a major cause of ubiquitin-positive frontotemporal lobar degeneration. Hum Mol Genet 2006; 15 : 2988-3001

19 Le Ber I, Camuzat A, Hannequin D et al: Phenotype variability in progranulin mutation carriers: a clinical, neuropsychological, imaging and genetic study. Brain 2008; 131: 732-746.

20 Masellis M, Momeni P, Meschino W et al: Novel splicing mutation in the progranulin gene causing familial corticobasal syndrome. Brain 2006; 129: 3115-3123.

21 Sleegers K, Brouwers N, Maurer-Stroh S et al: Progranulin genetic variability contributes to amyotrophic lateral sclerosis. Neurology 2008; 71: 253-259.

22 Schymick JC, Yang Y, Andersen PM et al: Progranulin mutations and amyotrophic lateral sclerosis or amyotrophic lateral sclerosis-frontotemporal dementia phenotypes. J Neurol Neurosurg Psychiatry 2007; 78: 754-756.

23 Chiang $\mathrm{HH}$, Rosvall L, Brohede J et al: Progranulin mutation causes frontotemporal dementia in the Swedish Karolinska family. Alzheimers Dement 2008; 4: 414-420.

24 Le Ber I, van der Zee J, Hannequin D et al: Progranulin null mutations in both sporadic and familial frontotemporal dementia. Hum Mutat 2007; 28: 846-855.

25 van der Zee J, Le Ber I, Maurer-Stroh S et al: Mutations other than null mutations producing a pathogenic loss of progranulin in frontotemporal dementia. Hum Mutat 2007; 28: 416.

26 Cairns NJ, Bigio EH, Mackenzie IR et al: Neuropathologic diagnostic and nosologic criteria for frontotemporal lobar degeneration: consensus of the Consortium for Frontotemporal Lobar Degeneration. Acta Neuropathol 2007; 114: 5-22.

27 Mackenzie IR, Neumann M, Baborie A et al: A harmonized classification system for FTLD-TDP pathology. Acta Neuropathol 2011; 122: 111-113.

28 Brouwers N, Sleegers K, Engelborghs S et al: Genetic variability in progranulin contributes to risk for clinically diagnosed Alzheimer disease. Neurology 2008; 71 : 656-664.

29 Shankaran SS, Capell A, Hruscha AT et al: Missense mutations in the progranulin gene linked to frontotemporal lobar degeneration with ubiquitin-immunoreactive inclusions reduce progranulin production and secretion. J Biol Chem 2008; 283 : 1744-1753.

30 Cruchaga C, Graff C, Chiang HH et al: Association of TMEM106B gene polymorphism with age at onset in granulin mutation carriers and plasma granulin protein levels. Arch Neurol 2011; 68: 581-586

31 Finch N, Carrasquillo MM, Baker M et al: TMEM106B regulates progranulin levels and the penetrance of FTLD in GRN mutation carriers. Neurology 2011; 76: 467-474.

32 Van Deerlin VM, Sleiman PM, Martinez-Lage M et al: Common variants at 7p21 are associated with frontotemporal lobar degeneration with TDP-43 inclusions. Nat Genet 2010; 42: 234-239.

33 Bernardi L, Maletta RG, Tomaino $\mathrm{C}$ et al: The effects of APOE and tau gene variability on risk of frontotemporal dementia. Neurobiol Aging 2006; 27: 702-709.

34 Farrer LA, Abraham CR, Volicer L et al: Allele epsilon 4 of apolipoprotein E shows a dose effect on age at onset of Pick disease. Exp Neurol 1995; 136: 162-170.

35 Minthon L, Hesse C, Sjogren M, Englund E, Gustafson L, Blennow K: The apolipoprotein E epsilon4 allele frequency is normal in fronto-temporal dementia, but correlates with age at onset of disease. Neurosci Lett 1997; 226: 65-67.

36 Pickering-Brown SM, Owen F, Isaacs A et al: Apolipoprotein E epsilon4 allele has no effect on age at onset or duration of disease in cases of frontotemporal dementia with pick- or microvacuolar-type histology. Exp Neurol 2000; 163: 452-456.

37 Hsiung GY, Fok A, Feldman HH, Rademakers R, Mackenzie IR: rs5848 polymorphism and serum progranulin level. I Neurol Sci 2010; 300: 28-32.

38 Rademakers R, Eriksen JL, Baker $\mathrm{M}$ et al: Common variation in the miR-659 bindingsite of GRN is a major risk factor for TDP43-positive frontotemporal dementia. Hum Mol Genet 2008; 17: 3631-3642.

Supplementary Information accompanies this paper on European Journal of Human Genetics website (http://www.nature.com/ejhg) 